doi:10.17659/01.2020.0056

Journal of Case Reports 2020;10(4):214-219

\title{
Primary Lymphoma of Long Bones causing Pathological Fracture: A Rare Entity
}

\author{
Swetabh Verma ${ }^{1}$, Saumitra Dwivedi ${ }^{1}$, Dharmendra Singh D ${ }^{1}$, Mohammad Zahid Khan ${ }^{1}$, Niti Singhal ${ }^{2}$ \\ Departments of ${ }^{1}$ Orthopaedics and ${ }^{2}$ Pathology, Bone and Joints Institute, Medanta, Lucknow, Uttar Pradesh, India.
}

\section{Corresponding Author:}

Dr Swetabh Verma

Email: swetabhverma26@gmail.com

This is an Open Access article distributed under the terms of the Creative Commons Attribution License (creativecommons.org/ licenses/by/3.0).

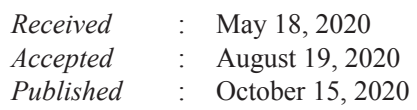

\begin{abstract}
Background: Non-Hodgkin's lymphoma ranks between $5^{\text {th }}$ to $9^{\text {th }}$ most $c o m m o n$ neoplasm worldwide, but the incidence of extra-nodal lymphoma is only between 3-5\%. Gastrointestinal system is the most common site of such extra-nodal involvement, those arising from bone are still a rare occurrence. Case Report: We present atypical manifestation of diffuse large B cell lymphoma of right proximal femur in a 75 year old gentleman. He presented to the outpatients clinic with a history of gradually progressive swelling in right groin and thigh since five months. Radiographs of right hip and thigh showed intra-medullary implants in situ with near total destruction of trochanteric region. Contrast tomography scans of right hip and thigh showed a circumferential lesion from trochanter till mid-thigh involving all the compartments. The biopsy from the lesion revealed diffuse large $\mathrm{B}$ cell lymphoma. The patient received chemotherapy and is in follow up with satisfactory response. Conclusion: Diffuse B cell lymphomas can be a rare diagnosis beyond $6^{\text {th }}$ decade and also present with pathological fractures with minimal constitutional symptoms and a slow growing mass. Strong clinical suspicion and prompt diagnosis can be useful in instituting an early chemotherapy.
\end{abstract}

Keywords: Diffuse Large B-Cell Lymphoma, Femur, Imaging, Magnetic Resonance Imaging. Thigh.

\section{Introduction}

Primary lymphoma of bone accounts for less than $5 \%$ of all bone tumors [1] and long bones of axial skeleton are the most common sites for it. The peak age of diagnosis is in the 50-60 year age group, being more common in men than in women. Symptoms are usually bone pain, sometimes swelling and rarely a pathological fracture. Most such cases are diagnosed with a single localized tumor. We present rare case of primary B cell lymphoma of right proximal femur in a 75 year old gentleman. Prior surgery for a fracture was sustained in subtrochanteric region five months ago. There was a history of trauma to same region twenty years ago with poor functional ability of right lower limb. This resulted in a delay at presentation to our outpatient clinic when the swelling at groin and thigh region had already assumed a massive size. A close observation and strong suspicion for such neoplasms must be kept in the background of persistent pain and slowly progressive swelling which can confound the identification of actual pathology.

\section{Case Report}

A 75 year old gentleman, moderately built and nourished, presented to our outpatient clinic with complaints of pain in the right groin and hip region with a swelling in the same area for last five months. He had not been bearing weight on the right lower limb since then. He also gave a history of intra-medullary implant following a fall and fracture of subtrochanteric region of right femur five months ago [Fig.1,2]. The patient was told at 


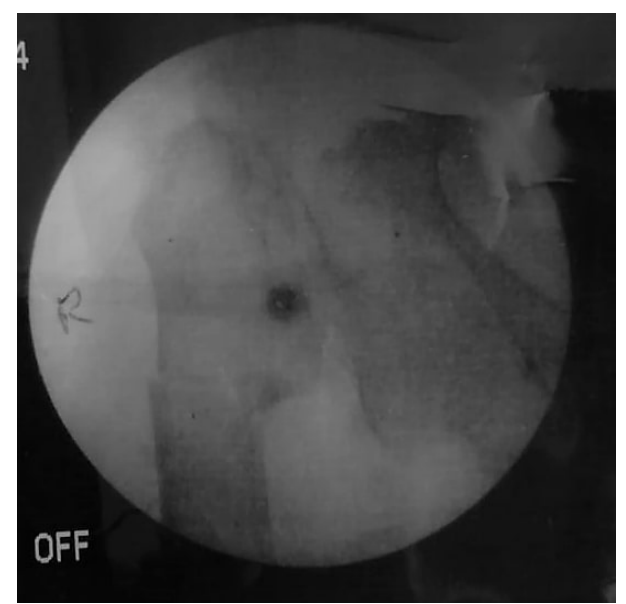

Fig.1: Pre-operative image prior to index surgery.

the time of index surgery that the fracture could be pathological and was advised further follow up and investigations. The pain in right hip region had persisted with minimal groin swelling at that time. On further enquiry, he also revealed that he had sustained trauma to the same region twenty years ago and had since then used a stick to aid walking till the recent episode of fracture.

At presentation, there was a swelling in right groin and upper thigh area measuring about $20 \times 20 \mathrm{~cm}$, firm in consistency with prominent veins [Fig.3-5]. There were three to four palpable lymph nodes in the inguinal region. There was no local rise of temperature or pulsations over the swelling. Distal neurovascularity of the limb was normal. His laboratory reports were largely within normal limits. There was no history of chronic addictions. Plain radiographs of hip and thigh showed near complete osteolysis of trochanteric region, intramedullary implants in situ and a soft tissue shadow over lateral aspect of hip region [Fig.6-8]. Due to presence of stainless steel implants, MRI of the right hip and thigh could not be done. Contrast CT scan of the same region showed a circumferential soft tissue mass extending from right trochanter till mid-thigh involving all the compartments and encroaching the muscle planes [Fig.9,10]. There were also enlarged external iliac and inguinal lymph nodes. A differential diagnosis of chondrosarcoma,
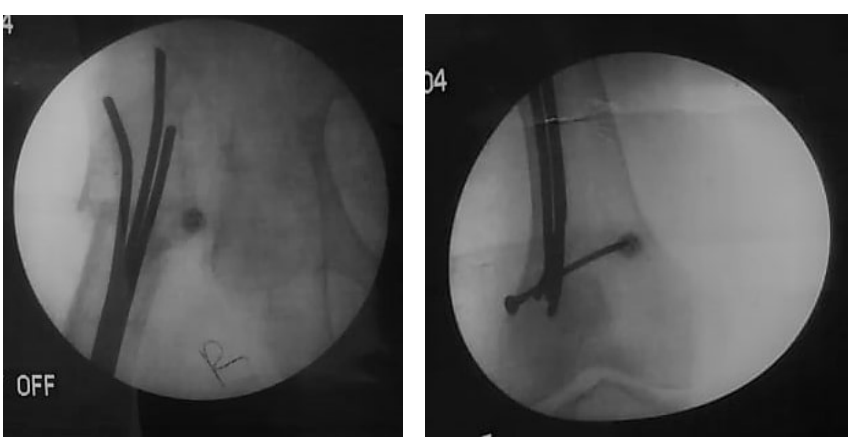

Fig.2: Post-operative image immediately after index surgery.
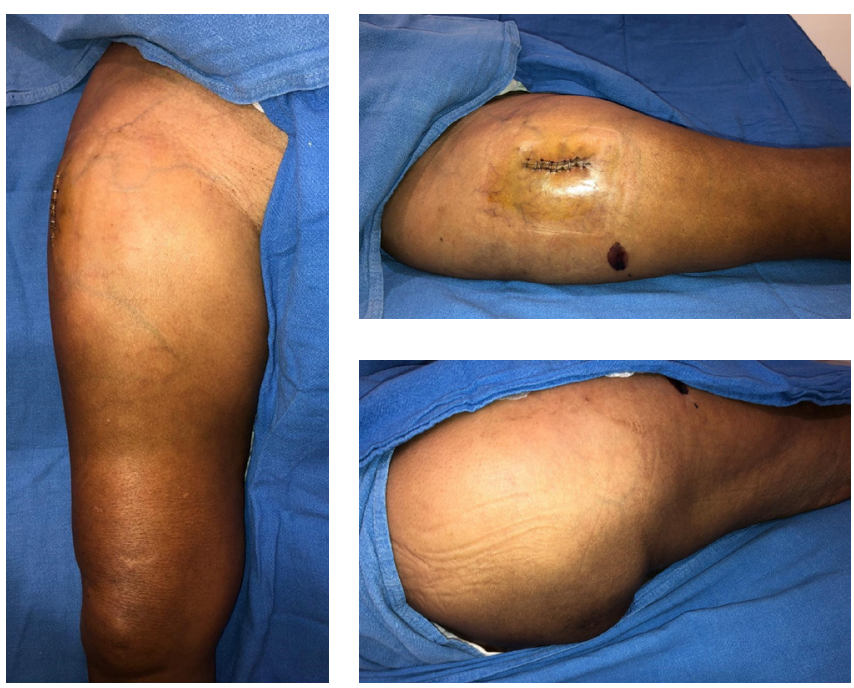

Fig.3-5: Clinical picture at presentation to us.

osteosarcoma and rhabdomyosarcoma was considered. Open biopsy of swelling was done for histological confirmation of diagnosis [Fig.11]. The excised tissue was of friable consistency and greyish-brown in color. Fine needle aspiration cytology specimens were also prepared. Postoperative period was uneventful. Fine needle aspiration cytology showed poorly differentiated round cell tumor and biopsy confirmed it to be diffuse large B cellnon-Hodgkin's lymphoma. There were sheets of atypical lymphoid cells with large vesicular nuclei, prominent nucleoli and frequent mitoses. The cells were positive for CD20 (B cell immunohistochemistry) [Fig.12]. The patient was referred to the hemato-oncology department where he was initiated on CHOP regimen and has been under follow up with satisfactory results. 


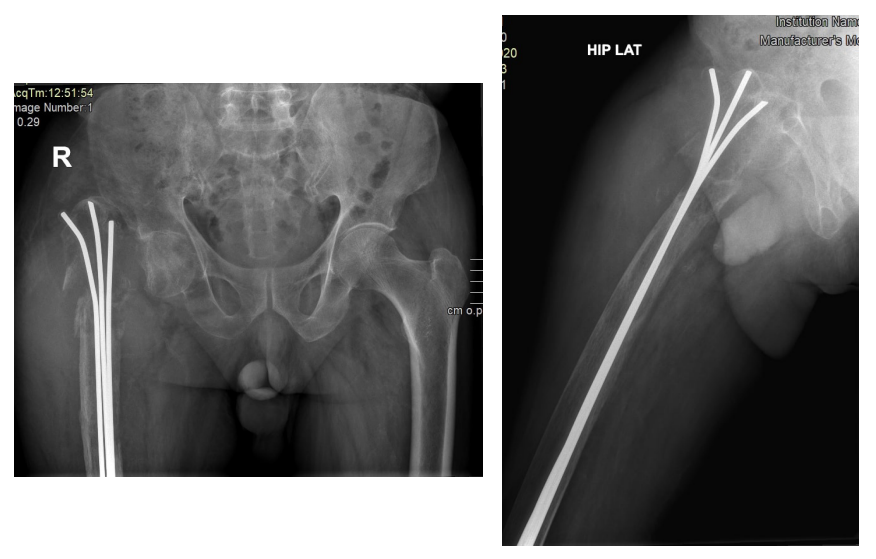

Fig.6,7: Plain and lateral radiograph at time of presentation to our hospital.

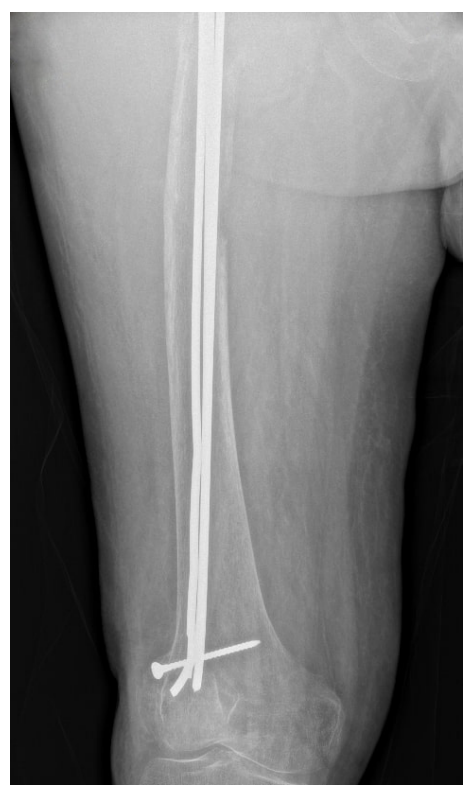

Fig.8: Plain radiograph of knee with thigh at presentation.

\section{Discussion}

Non-Hodgkin's lymphoma (NHL) is a group of neoplastic lesions arising out of lymphoid tissue, either from lymph nodes or with an extra-nodal origin. Triggered by an uncontrolled proliferation of B or T cell lymphocytes, NHL is further subclassified into ten types based on aggressiveness, morphology and response to treatment. Based on survival characteristics, it is convenient to broadly identify them as either low grade, intermediate grade or high grade.
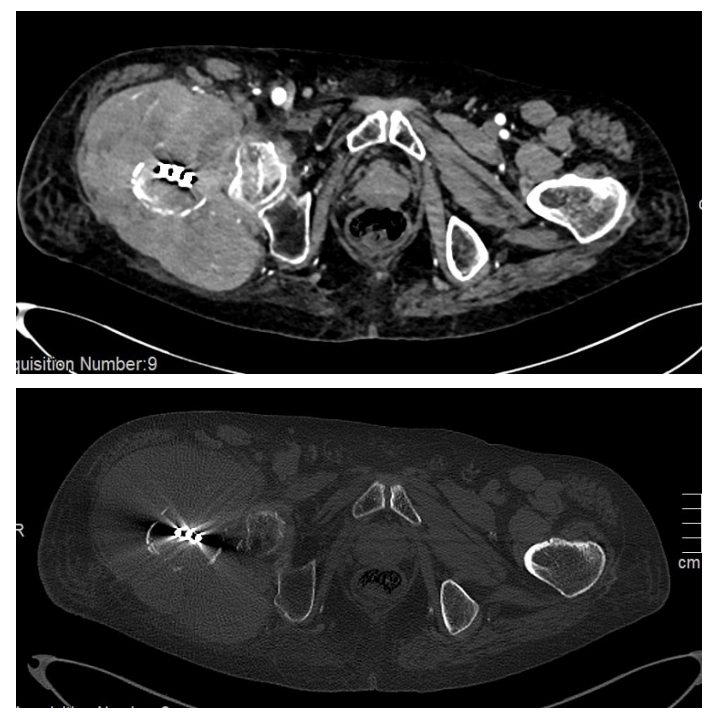

Fig.9,10: Axial contrast CT section.

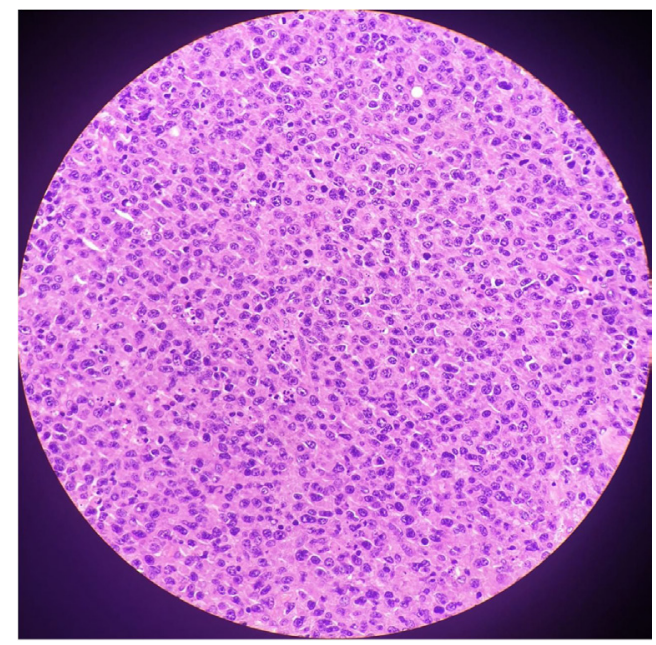

Fig.11: Histopathology slides.

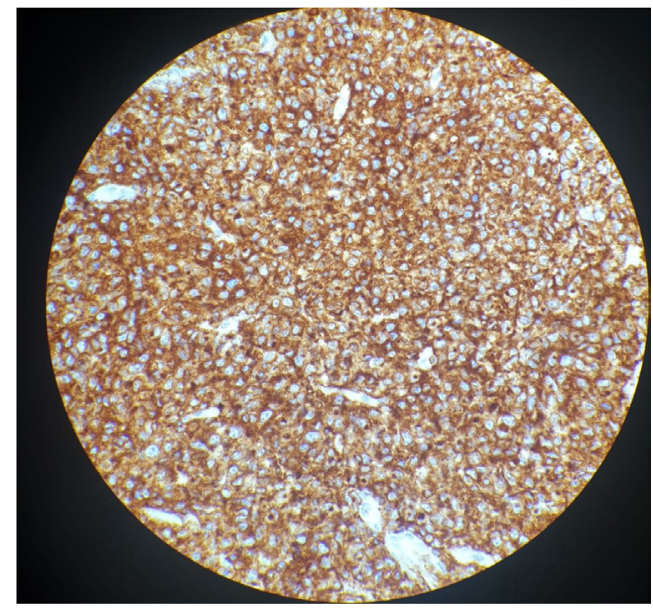

Fig.12: Histopathology slides with immunohistochemistry. 
Infiltration of rapidly proliferating lymphoid cells into organs other than lymph nodes is called extra-nodal involvement. Most common area of involvement is gastrointestinal system (stomach being the commonest), followed by Waldeyer's ring in the nasooropharynx, lung, liver, spleen and bones [2]. Origin of tumor primarily outside lymph nodes is called primary extra-nodal lymphoma. Spread of malignant lymphoid cells via hematogenous medium to extra-nodal sites from the lymph nodes is called secondary extra-nodal lymphoma. Diffuse large B cell lymphoma is a rapidly growing, aggressive form of NHL.

Primary lymphomas of bone and muscles is a relatively rare entity and most of them are NHL with a very few being Hodgkin's disease. Primary bone NHL accounts for less than $2 \%$ of adult lymphomas and around $3 \%$ of primary bone tumors [3]. Though the origin of these lymphomas has largely remained a matter of debate, chromosomal aberrations, translocations have been frequently postulated for the same. Recently, the role of Epstein Barr virus (EBV), Human Immunodeficiency virus (HIV) and Human Herpes virus-6 (HHV-6) have been postulated but hasn't been confirmed so far [4].

Most common site for it is long bones of axial skeleton, being femur and tibia when the involvement is monoostotic. Other reported sites have been vertebral column (when the involvement is polyostotic), pelvis and rarely ribs, clavicle and scapula [5]. There is a slight male predominance and usually presents after $5^{\text {th }}$ or $6^{\text {th }}$ decade. Rare case of occurrence in proximal phalanx of thumb has also been reported [6]. In the younger patients, the differential diagnosis can be osteosarcoma, Ewing's sarcoma, and osteomyelitis. In the older patients, bone metastasis of the solid tumors should be considered. The study of epidemiology of Non-Hodgkin's lymphoma in Indian population found a high incidence of B symptoms (40-60\%) [7]. However, these symptoms were conspicuously absent in our case. This study also identified that diagnostic and treatment delays, incorrect diagnosis and inappropriate or suboptimal treatment as possible reasons for the poor outcome.

Lymphomas which present primarily at femur, tibia or proximal humerus with pathological fractures are unusual. The pathological fracture is due to either bone damage by the tumor, radiotherapy, or disuse atrophy and usually presents after many years of diagnosis of lymphoma. In a large series of 131 patients by Ramadan KM et al. [8], only nine patients presented with a pathological fracture. However, we observed that in our case, the patient was brought primarily for treatment of pathological fracture at an elsewhere facility when the underlying diagnosis of lymphoma was not confirmed. Only after the initial fracture stabilization, when there was a gradual appearance of swelling within a period of five months that he presented to our hospital for further management.

Two types of radiological imaging patterns have been identified in primary bone lymphomas by Krishnan A et al. [9]. Most commonly, it's a 'lytic destructive pattern' seen in $70 \%$ cases with remaining being 'blastic sclerotic type'. An associated soft tissue mass indicates a poorer prognosis. Our case was consistent with the 'lytic destructive' pattern with a large lytic lesion at trochanteric region, a permeative pattern which extended into the metaphysis, a cortical breakthrough and a pathological fracture. The 'blastic sclerotic' pattern is more commonly seen in patients with metastasis.

The histological findings of primary NHL of the bone mimic the conventional, non-osseous forms of NHL. The hallmark feature is a replacement of the normal marrow constituents by neoplastic lymphoid tissue [10]. Coley BL et al. have laid down criteria [11] for diagnosis of such primary lymphomas of bone which requires a primary focus in a single bone, a positive histological 
diagnosis and a lack of evidence of a distal soft tissue or a nodal involvement. The Coley's criteria was also fulfilled in our case, further supporting the diagnosis.

Plain radiograph, though most commonly used as initial assessment tool, is largely insufficient to provide information on extent of tumor invasion and compartment encroachments. Though MRI is the most sensitive imaging technique which can be used for the diagnosis of primary bone lymphomas, due to presence of metallic intramedullary implants, we had to replace it with contrast CT scan. The role of PET scans has not been extensively studied in such lesions due to its rarity, but it stands ahead of MRI in assessing the remission status.

The treatment of primary lymphomas of bone is largely chemotherapy, most frequently using the CHOP regimen (cyclophospahamide, hydroxydaunorubicin, oncovin and prednisolone) for six to eight cycles. The role of surgery is restricted to biopsy and management of pathological fractures. Radiotherapy is not a preferred modality either alone or in combination with chemotherapy due to high incidence of postradiotherapy sarcomas $[8,12]$.

Though our case was in agreement with most of the published literatures on primary bone lymphomas, however it could not be ascertained as to whether the occurrence of pathological fractures draws the attention to primary diagnosis. As we did not have any positive history from the patient prior to five months, we are uncertain if the patient had a low grade lymphoma prior to that. A short follow up period of six months is also a limiting factor for this case.

\section{Conclusion}

In Indian scenario, where the initial symptom of pain may be overlooked by the patients, presentation to emergency department may be due to pathological fractures. A strong index of clinical suspicion of non-Hodgkin lymphoma must be kept in male patients beyond sixth decade with fragility fractures of proximal femur, tibia or humerus and must be thoroughly investigated before attempting any surgical intervention for fracture stabilization.

Contributors: SV: manuscript writing original draft preparation, patient management; SD, DS, MZK: manuscript revision and patient management. NS: critical inputs into the manuscript and histopathology. SV will act as study guarantor. All authors approved the final version of the manuscript and are responsible for all aspects of the study.

Funding: None; Competing interests: None stated.

\section{References}

1. Jain A, Alam K, Maheshwari V, Khan R, Nobin H, Narula V. Primary bone lymphomas - Clinical cases and review of literature. J Bone Oncol. 2013;2:132-136.

2. Paes FM, Kalkanis DG, Sideras PA, Serafini AN. FDG PET/CT of extranodal involvement in non-Hodgkin lymphoma and Hodgkin disease. Radiographics. 2010;30:269-291.

3. DG, McVey GP, Korpanty G, Treacy A, Dervan P. O'Keane C, et al. Primary bone lymphoma: single institution case series. Ir. J Med Sci. 2008;177:247-251.

4. Swerdlow SH, Campo E, Harris NL, Jeffe ES, Pileri $\mathrm{SA}$, Stein $\mathrm{H}$, et al. WHO classification of tumours of haematopoietic and lymphoid tissues. $4^{\text {th }}$ ed. Geneva, Switzerland: IARC Press.2008.

5. Messina C, Ferreri AJM, Govi S, Bruno-Ventre M, Medina EAG, Porter D, et al. Clinical features, management and prognosis of multifocal primary bone lymphoma: a retrospective study of the international extranodal lymphoma study group (the IELSG 14 study). Br J Haematol. 2014;164:834-840.

6. Pinheiro RF, Filho FD, Lima GG, Ferreira FV. Primary non-Hodgkin lymphoma of bone: An unusual presentation. Instituto do Cancer do Ceará. J Cancer Res Ther. 2009;5:52-53.

7. Nair R, Arora N, Mallath MK. Epidemiology of NonHodgkin's lymphoma in India. Oncology. 2016;9:18-25.

8. Ramadan KM, Shenkier T, Sehn LH, Gascoyne RD, Connors JM. A clinicopathological retrospective study on 131 patients with primary bone lymphoma: A population-based study of successively treated cohorts from the British Columbia Cancer Agency. Ann Oncol. 2007;18:129-135.

9. Krishan A, Shirkoda A, Tehranzadeh J, Armin AR, Irwin $\mathrm{R}$, Les K. Primary bone lymphoma: A radiographic-MR 
imaging correlation. Radiographics. 2003;23:13711387.

10. Singh $T$, Lakshmaiah CT, Suresh TM, Babu GK, Lokanatha D, Jacob LA, et al. Primary bone lymphoma: A report of two cases and review of the literature. J Can Res Ther. 2010;6:296-298.

11. Coley BL, Higginbotham NL, Groesbeck HP. Primary reticulum cell sarcoma of the bone; a summary of 37 cases. Radiology. 1950;55:641-658.

12. Ibrahim I, Bryan D. Haughom BD, Fillingham Y, Gitelis S. Is radiation necessary for treatment of non-Hodgkin's lymphoma of bone? Clinical results with contemporary therapy. Clinical Orthopaedics and Related Research. 2016;474:719-730. 